\title{
Measurement of Error in Electrostriction Based Dielectrics
}

\author{
Anjani Kumar Singh \\ Bharati Vidyapeeth's College of Engineering, New Delhi, India \\ Email: anjaninsit@gmail.com
}

Received 1 April 2014; revised 5 May 2014; accepted 15 May 2014

Copyright (C) 2014 by author and OALib.

This work is licensed under the Creative Commons Attribution International License (CC BY). http://creativecommons.org/licenses/by/4.0/

(c) (i) Open Access

\begin{abstract}
While correlating the various components of mechanical stress tensor due to elastic response with the corresponding components of electrically induced stress tensor pertaining to quadratic electrostriction, proper precautions are to be observed for higher order electromechanical coupling. Contributions from lateral stresses, electrical and mechanical boundary conditions are to be considered for the correct estimation of induced strain in elastic dielectrics. The knowledge of dependence of Maxwell's electrostatic stresses on dielectric constants and on the orientation of dielectric material with respect to the electric field vector is necessary for the exact estimation of electrically induced strains. The contributions from the variation in transverse components of dielectric tensor produced by the variation in lateral stresses are to be incorporated into the expression for the correct estimation of electrostrictive coefficients. The electromechanical behavior of elastic dielectric is discussed and the errors often committed in using incorrect formulae for electrostriction are reported.
\end{abstract}

\section{Keywords}

Elastic Dielectric, Electrostriction, Induced Strain, Lateral and Shear Stresses

\section{Introduction}

The analysis that has been presented in this paper gives a guideline indicating errors committed in the estimation of the electrically induced strain in elastic dielectrics. The Maxwell stress effect occurs due to variation in electric field distribution with strain and the phenomena of electrostriction occur due to variation in dielectric properties of the material with strain. The linear elastic response governed by Hooke's law, the Maxwell electrostatic stress governed by coulomb's law and the electrostriction stress (dielectric response) are illustrated in Figure 1.

The deformed material (generally non piezoelectric) is no longer isotropic, and the scalar permittivity $(\varepsilon)$ becomes dielectric tensor $\left(\varepsilon_{i j}\right)$ due to its anisotropy behavior. 


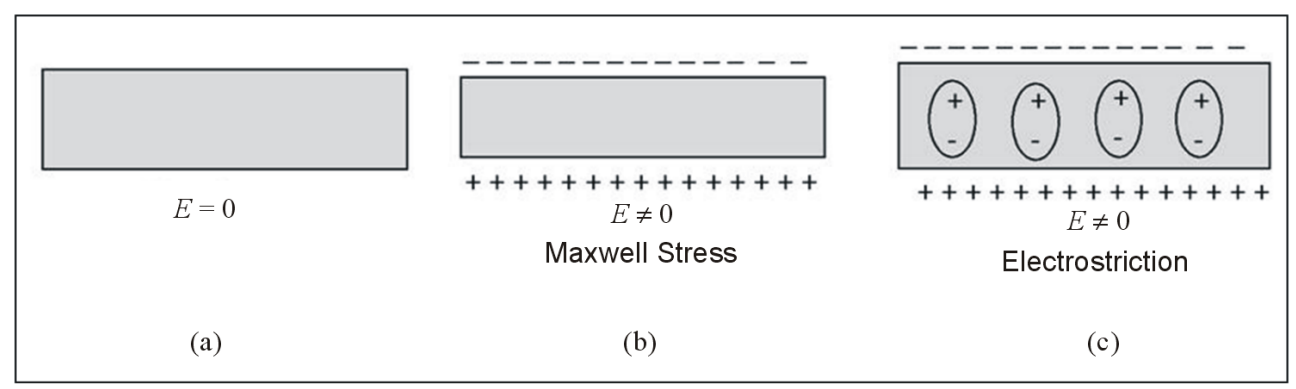

Figure 1. Schematic diagrams illustrating (a) pure linear elastic response governed by Hooke's law for $E=0$ (b) Maxwell stress produced by the surface charge and governed by coulomb law for $E \neq 0 \quad \&$ (c) Electrostriction stress produced due to alignment of dipoles inside the dielectric material for $E \neq 0$.

As the deformation is extremely small, only the first order terms in strain tensor $S_{i j}$ have been considered and the dielectric tensor $\varepsilon_{i j}$ in terms of the strain tensor is given [1] by

$$
\varepsilon_{i j}=\varepsilon^{0} \delta_{i j}+a_{1} S_{i j}+a_{2} S_{k k} \delta_{i j},
$$

where $\varepsilon^{0}$ is the permittivity of the undeformed body and $a_{1} \& a_{2}$ are two parameters describing the variation in dielectric properties of the material in shear and bulk deformation respectively.

The Maxwell stress tensor [1], derived on the fundamental assumptions for the linear electrostriction, is a result of force produced by the electric field and is given as

$$
T_{i j}=T_{i j}^{0}+\frac{\varepsilon_{0}}{2}\left(2 \varepsilon^{0}-a_{1}\right) E_{i} E_{j}-\frac{\varepsilon_{0}}{2}\left(\varepsilon^{0}+a_{2}\right) E^{2} \delta_{i j},
$$

where $T_{i j}^{0}$ is stress tensor in the absence of an external electric field. Generally we use to neglect $T_{i j}^{0}$ in isotropic dielectrics but in case of composite dielectric material or dielectric interface, the presence of significant number of point defects introduces distortion. In case of size difference of two particles from two phases, elastic stress strains are created. A larger atom introduces compressive stress and corresponding strain around it, while a smaller interacting atom creates a tensile stress-strain field. An interstitial atom also produces strain around the void it is occupying. However, it is very difficult to evaluate $T_{i j}^{0}$ precisely [2].

$$
S_{i j}=\frac{1}{Y}\left[(1+\sigma) T_{i j}-\sigma T_{k k} \delta_{i j}\right],
$$

conversely,

$$
T_{i j}=\frac{Y}{1+\sigma}\left(S_{i j}+\frac{\sigma}{1-2 \sigma} S_{k k} \delta_{i j}\right) .
$$

It is very important to note that the above Equations (2) and (4) have been correlated in the electromechanical coupling on taking certain approximations, however these equations have been formulated certainly on different assumptions particularly in respect of range of interaction for the body forces and surface forces in continuum mechanics, and polarization effect in dielectrics. For the measurement of electrically induced strains, the dependence of traction vector (at the boundary/interface between the dielectric material and electrodes) on the orientation of dielectric sample with the electric field is of practical interest [3]. However the boundary conditions [4] at the interface between electrode and the dielectrics are the points of main concern as it is very difficult to predict exact mechanical and electrical boundary conditions in the deformed dielectrics.

The objective of this work is to provide a clear understanding of the possible mechanism involving various dielectric and mechanical parameters for electric field induced strains in elastic dielectrics. Experimental data on the subject are not abundant and the researchers [5] [6] frequently used incorrect formulae in the derivation of elastic strain with respect to the Maxwell stress effect, particularly in case of polyurethane elastomer (a crossed linked polymer), assuming various unrealistic approximations as observed by [7]. A linear electromechanical effect does not exist in case of elastomers and the Hooke's law based on thermodynamics consideration (Helmoholtz free energy and Gibbs free energy concept) should not be applied for elastomers up to a large extent 
due to its nonlinear elastic behavior. Electromechanical coupling effects in case of non-piezoelectric material such as polyurethane elastomers have been exploited in the areas of fundamental sensors and actuators [8] [9]. Due to potential applications in sensing and actuation, the electrostriction response is very significant [10].

\section{Maxwell Electrostatic Stresses}

In electromagnetic field theory, the Maxwell stress tensor $T_{i j}$ has been formulated [11] using classical electrostatic and assuming uniform electric field as

$$
\begin{aligned}
T_{i j} & =E_{i} D_{j}-\frac{1}{2} \delta_{i j} \sum_{k=1}^{3} E_{k} D_{k} \\
& =E_{i} \sum_{k=1}^{3} \varepsilon_{j k} E_{k}-\frac{1}{2} \delta_{i j} \sum_{m=1}^{3} \sum_{k=1}^{3} \varepsilon_{m k} E_{k} E_{m}
\end{aligned}
$$

where $E_{i}$ is the electric field strength vector, $D_{i}$ is the electric displacement vector, and $\varepsilon_{i j}$ is the dielectric tensor. A symmetric dielectric tensor $\varepsilon_{i j}$ in case of triclinic crystal is given by

$$
\varepsilon_{i j}=\left[\begin{array}{lll}
\varepsilon_{11} & \varepsilon_{12} & \varepsilon_{13} \\
\varepsilon_{21} & \varepsilon_{22} & \varepsilon_{23} \\
\varepsilon_{31} & \varepsilon_{32} & \varepsilon_{33}
\end{array}\right]
$$

If an electric field is parallel to the Z-axis (unidirectional field as in case of parallel plate capacitor), the tensor $T_{i j}$ is reduced [3] to

$$
\begin{aligned}
T_{i j}\left(0,0, E_{z}\right) & =\left[\begin{array}{ccc}
-\frac{1}{2} \varepsilon_{33} E_{z}^{2} & 0 & 0 \\
0 & -\frac{1}{2} \varepsilon_{33} E_{z}^{2} & 0 \\
\varepsilon_{13} E_{z}^{2} & \varepsilon_{23} E_{z}^{2} & \frac{1}{2} \varepsilon_{33} E_{z}^{2}
\end{array}\right] \\
& =\left[\begin{array}{ccc}
0 & 0 & 0 \\
0 & 0 & 0 \\
\varepsilon_{13} E_{z}^{2} & \varepsilon_{23} E_{z}^{2} & \frac{1}{2} \varepsilon_{33} E_{z}^{2}
\end{array}\right]-\frac{1}{2} \varepsilon_{33} E_{z}^{2}\left[\begin{array}{ccc}
1 & 0 & 0 \\
0 & 1 & 0 \\
0 & 0 & 1
\end{array}\right]
\end{aligned}
$$

the first term of this equation represents tension along $\mathrm{X} \& \mathrm{Y}$ direction, the second term implies a compression along Z-direction. Generally compression along longitudinal axis (Z-axis) is always accompanied by extension along transverse plane (X-Y plane) as in (Figure 2) and vice-versa. It is also clear from the above Equation (7) that the stress tensor with electric field in Z-direction has affected only the components $\varepsilon_{33}, \varepsilon_{13} \& \varepsilon_{23}$ of dielectric tensor $\varepsilon_{i j}$ corresponding to the strain component $S_{33}, S_{13} \& S_{23}$ respectively and other components become zero. The lateral strains $\left(S_{13} \& S_{23}\right)$ exist [11] in the capacitor electrode even if the dielectric medium is empty space. So the contribution from lateral strains should not be neglected for the correct estimation of results.

\section{Coupling of Dielectric and Elastic Response Parameters}

The direction of electric field is assumed to be along Z-axis $\left(E_{z} \neq 0, E_{x}=E_{y}=0\right)$ in the cartesian co-ordinate system. For the mechanically isotropic material $\left(T_{i j}^{0}=0\right)$, the electrically induced stresses given by Equation (2) become

$$
\begin{aligned}
& T_{33}=+\frac{1}{2} \varepsilon_{0} \varepsilon^{0} E^{2}\left(1-\frac{a_{1}+a_{2}}{\varepsilon^{0}}\right), \\
& T_{11}=T_{22}=-\frac{1}{2} \varepsilon_{0} \varepsilon^{0} E^{2}\left(1+\frac{a_{2}}{\varepsilon^{0}}\right),
\end{aligned}
$$




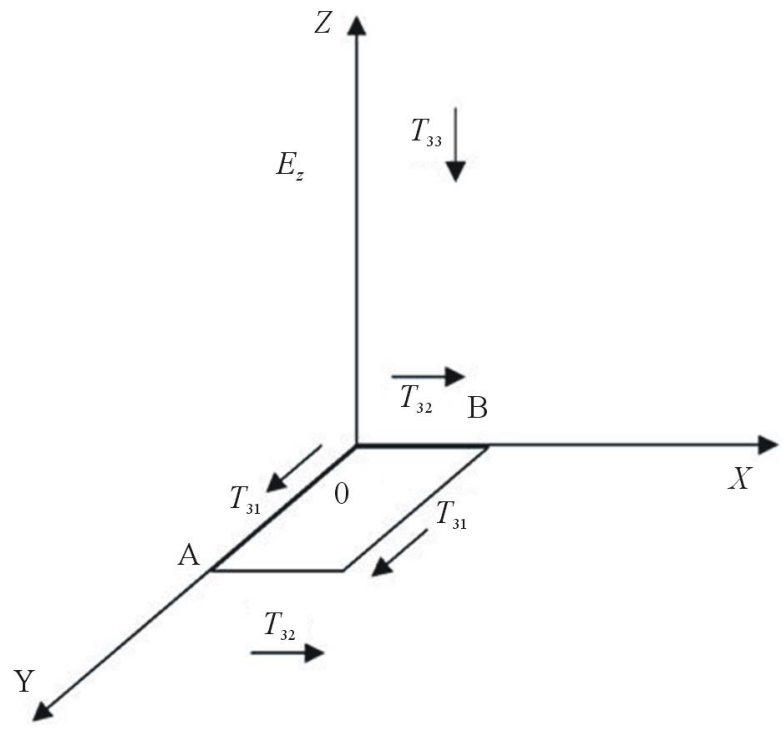

Figure 2. Schematic diagram indicating compression due to normal stress along Z-directions and is accompanied by the extension due to shear stresses along $\mathrm{X}-\mathrm{Y}$ plane.

$$
T_{13}=T_{23}=0 .
$$

Equation (8) represents tension along longitudinal (Z-axis) axis and the Equation (9) represents compression along transverse plane. However the researchers [5] [7] [12] have indicated the opposite characteristic of Equations (8) and (9).

The electrically induced stresses will generate elastic strains in equilibrium condition by the method of superposition. On putting the principal value of Maxwell stress Tensor from Equation (8) and Equation (9) into the corresponding components of Equation (3), we get Sacerdote's formula for the relative change in thickness of an elastic dielectric material as the coupling expressions between two phenomena and are given by

$$
\begin{aligned}
& S_{11}=S_{22}=-\frac{1}{2} \varepsilon_{0} \varepsilon^{0} E_{z}^{2}\left[1-\frac{\sigma a_{1}}{\varepsilon^{0}}+\frac{(1-2 \sigma) a_{2}}{\varepsilon^{0}}\right] Y^{-1}, \\
& S_{33}=+\frac{1}{2} \varepsilon_{0} \varepsilon^{0} E_{z}^{2}\left[(1+2 \sigma)-\frac{a_{1}+(1-2 \sigma) a_{2}}{\varepsilon^{0}}\right] Y^{-1}
\end{aligned}
$$

In unilateral mechanical deformations described in continuum mechanics, the components of elastic stress tensor (elastic response) of Equation (4) have been correlated with the corresponding components of electrostriction stress tensor (dielectric response) of Equation (2). Sufficient precautions have to be taken while coupling the dielectric response (represented by Equation (2)) with the elastic response (represented by Equations (3) and (4)) to derive the Equations (11) and (12) and correlating higher order components of two phenomena [11]. Because assumptions made for the derivation of mechanical stress tensor (Equation (4)) and electrostriction stress tensor (Equation (2)) are almost inconsistent and both of them can be endowed with a different physical meaning [13]. It has also been pointed out [14] [15] that the body forces (described by a body force density) and the surface force (described by a stress tensor) cannot be applied to all continuous media.

The principal elastic strain, $S_{33}$, represents the relative change in thickness of dielectric slab where as the principal strains, $S_{11}$ or $S_{22}$, represent the relative change in diameter of the capacitor as described by [7] which is not correct in case of a parallel plate capacitor in which $S_{31} \& S_{32}$ actually represents the lateral change in dimension as the electric field $E$ has only one component along $\mathrm{Z}$ direction (Figure 3) i.e. only one normal component of stress, $T_{33}$ along the direction of electric field and two shearing components of stress tensor, $T_{31} \& T_{32}$ along X-Y plane.

On comparing Equations (11) \& (12) with the general equation, $S_{i j}=\gamma_{i j k l} E_{k} E_{l}$, for electrostriction, we get 


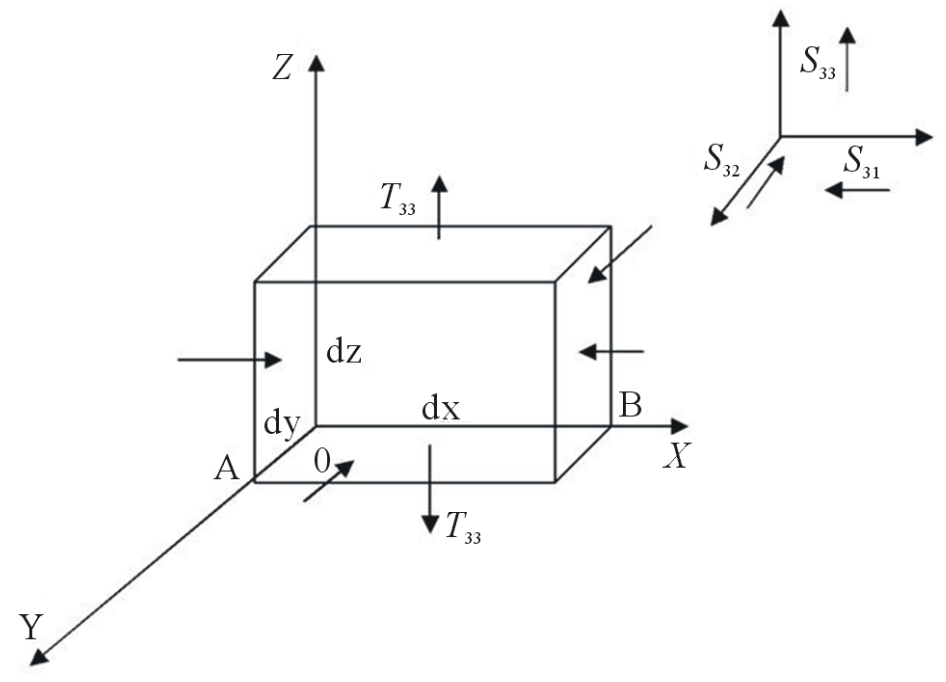

Figure 3. Schematic diagram illustrating extension due to electrostriction stress tensor along Z-direction and compression due to shear stress tensor along X-Y plane.

$$
S_{11}=S_{22}=-\gamma_{31} E^{2}, S_{33}=+\gamma_{33} E^{2}
$$

where $\gamma_{31} \& \gamma_{33}$ are electrostrictive coefficients. The negative sign for strains $S_{11} \& S_{22}$ indicates contraction along $\mathrm{X} \& \mathrm{Y}$ direction, and the positive sign for $S_{33}$ indicates expansion along $\mathrm{Z}$ direction as indicated in Figure 3.

If the electric field $E$ is changed to $E+\Delta E$, then the change in $S_{33}$ becomes,

$$
\Delta S_{33}=+2 \lambda_{33} E_{0} \Delta E=\mu_{33} \Delta E,
$$

where $\mu_{33}$ is a piezoelectric coefficient. Similarly, the coefficient $\mu_{31} \& \mu_{32}$ can be found along X \& Y directions. The piezoelectric transverse coefficients have been frequently used [16] in piezoelectric thin film devices. The average value of compressive elastic strain induced along Z-direction is

$$
K \int_{0}^{d} \Delta S_{33} \mathrm{~d} z
$$

where $d$ is the longitudinal thickness of elastic dielectric slab. Similarly, the expression for the compressive strains $\left(\Delta S_{32} \& \Delta S_{31}\right)$ along $\mathrm{X} \& \mathrm{Y}$ direction can be obtained. For nonuniform field as in case of cylindrical capacitor or interface, average value of $E^{2}$ can be calculated as

$$
\overline{E^{2}}=\frac{1}{d} \int_{0}^{d} E^{2}(z) \mathrm{d} z,
$$

where the integration is over the thickness $d$ of dielectric slab of a capacitor. In the presence of a non-uniform electric field with high magnitude, the phenomenon of dielectrophoresis [4] takes place, however it is in general a weak effect particularly in case of a solid dielectric.

\section{Measurement of Electrostrictive Coefficients and the Induced Strain}

On differentiating Equation (1) with respect to $S_{33}$, we get

$$
\frac{\mathrm{d} \varepsilon_{33}}{\mathrm{~d} S_{33}}=a_{1}+a_{2} \frac{\mathrm{d} S_{k k}}{\mathrm{~d} S_{33}}=a_{1}+a_{2}(1-2 \sigma),
$$

Assuming, if the normal force is acting only along $\mathrm{Z}$ direction i.e. along the direction of electric field $E$, then we have $S_{11}=S_{22}=0$ and if the sides of dielectric material are also fixed by the rigid wall (Figure 4) then we have $S_{32}=S_{31}=0$. We have, for the constrained situations as in Figure 4, 


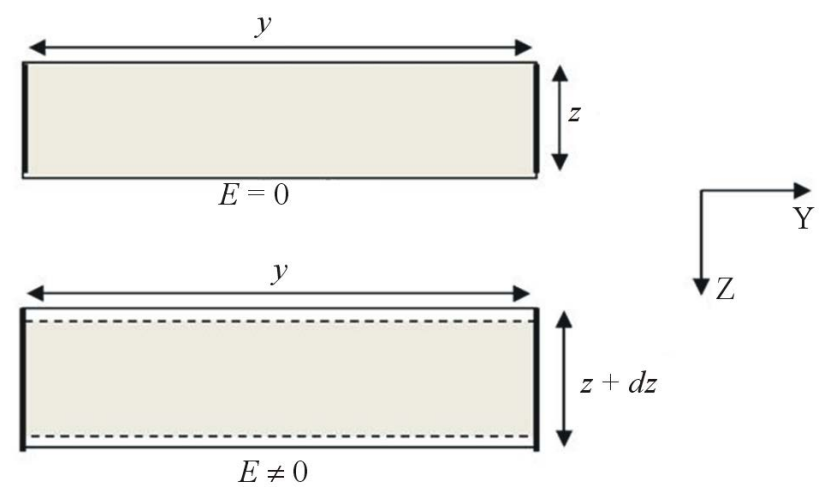

Figure 4. Schematic diagrams illustrating dielectric sample with side of capacitor rigid and fixed (in a constrained situation with $S_{32}=S_{31}=0$ ) for (a) $E=0$ \& (b) $E \neq 0$.

$$
\frac{\mathrm{d} \varepsilon_{33}}{\mathrm{~d} S_{33}}=a_{1}+a_{2} .
$$

The second term under bracket of Sacerdate formula Equation (12) can be compared with Equation (17) and the Equation (12) is given as

$$
S_{33}=\frac{\nabla z}{Z}=-\frac{1}{2} \varepsilon_{0} \varepsilon^{0} E^{2}\left[(1+2 \sigma)-\frac{1}{\varepsilon_{0}} \frac{\mathrm{d} \varepsilon_{33}}{\mathrm{~d} S_{33}}\right] .
$$

Several researchers [5] [6] have measured second term under bracket by various methods.

For a parallel plate capacitor, the capacity is

$$
C=\frac{\varepsilon_{0} \varepsilon^{0} A}{h},
$$

where $A$ is area of plate, $h$ is separation between plates, $\varepsilon^{0}$ is permittivity. Differentiating above equation, we get

$$
\mathrm{d} C=\frac{\varepsilon_{0} A}{h} \mathrm{~d} \varepsilon^{0}+\frac{\varepsilon_{0} \varepsilon^{0}}{h} \mathrm{~d} A-\left(\varepsilon_{0} \varepsilon^{0} A\right) \mathrm{d} h
$$

From (20) \& (21), we get

$$
\frac{\mathrm{d} C}{C}=\frac{\mathrm{d} \varepsilon^{0}}{\varepsilon^{0}}+\frac{\mathrm{d} A}{A}-\frac{\mathrm{d} h}{h}
$$

If the electrodes are rigid and incompressible, then $\mathrm{d} A=0$ and Equation (22) becomes

and

$$
\left(\frac{\mathrm{d} C}{C}\right)_{\text {die }}=\frac{\mathrm{d} \varepsilon^{0}}{\varepsilon^{0}}-\frac{\mathrm{d} h}{h},
$$

From Equation (19), the electrostrictive term is

$$
\frac{1}{\varepsilon_{0}} \frac{\mathrm{d} \varepsilon_{33}}{\mathrm{~d} S_{33}}=\frac{\mathrm{d} \varepsilon_{33} / \varepsilon_{0}}{\mathrm{~d} h / h} .
$$

The relative change in thickness and the dielectric parameters, $a_{1} \& a_{2}$, are experimentally obtained [17] by determining $\mathrm{d} \varepsilon_{33} / \mathrm{d} S_{33}$ in constrained and unconstrained situation, with and without dielectric material, in a capacitor. However the linearity of the theory for electrostriction fails if the deformations are not quadratic in the applied field particularly at higher field strength. But very few reliable measurements of electrostrictive 
coefficients $\left(a_{1} \& a_{2}\right)$ for solids have been reported in the literature.

\section{Discussion and Comparison with Previous Models: Errors in Estimation}

Errors are often committed in the derivation of suitable expression for the interpretation of experimental results. The above expressions (Equations (23)-(25)) have been derived on the sole assumption that permittivity $\varepsilon_{33}^{0}$ changes with longitudinal strain only but the permittivity also changes with respect to the lateral strain/stress in the capacitor depending on the crystal structure of elastic dielectric medium. In triclinic, monoclinic \& orthorhombic crystal systems, all the three principal values of permittivity are different, whereas, in the cubic system, these values are same [1]. So the correction due to lateral strain \& corresponding change in lateral permittivities $\varepsilon_{13} \& \varepsilon_{23}$ (Equation (6)) must be incorporated to get correct mathematical modeling. The researcher [17] has stopped the change in lateral strain by considering constrained situation (considering sides of capacitor fixed \& rigid) but fail to understand that the change in lateral permittivity is also due to lateral electrostatic stresses (Equation (7)).

\subsection{Shear Stresses}

Zhang et al. [6] use non-tensor form of stresses (T) and strains (S) for experimental determination of induced strains in the case of Polyurethane elastomers (DOW 2103-80AE) and the relative change in thickness of the dielectric slab of a parallel plate capacitor is considered as

$$
S_{33}=-\frac{1}{2 Y} \varepsilon_{0} \varepsilon^{0} E_{z}^{2}
$$

which is quite different from the Equation (12) particularly in case of an elastomer or an elastic dielectric material with large Poisson's ratio $(\sigma \approx 0.5)$. So the neglect of shear stresses/strains leads to incorrect estimation [7] of induced strain. Similarly, another researcher [5] used Equation (8) without considering contributions from shear stresses, edge effect and orientation of electric field with respect to sample.

Errors in the estimations of induced strain can be pointed out for the case of Polycarbonate (PC). The following data for Polycarbonate are given [17] as:

$$
\left|a_{1}\right|=1.404,\left|a_{2}\right|=2.6 \text {. }
$$

Substituting above data in Equations (12) and (26), an overestimation 179\% has been found. Similarly comparing the Equation (8) divided by $Y$ as considered by [5] with Equation (12), an overestimation of $137 \%$ has been observed.

The use of correct Equation (12) is necessary particularly for the material having high value of Poisson's ratio. Using dielectric constant and the elastic compliance data the contribution of the Maxwell stress to the total strain response can be determined. For accurate estimation of induced strains, the contributions from coordinate axis or orientation (of sample with respect to the normal principal stress) dependent of Young modulus, Poisson's ratio and generalized moduli should also be incorporated into the expressions. A comprehensive investigation and computations [18] carried out for BCC crystal under hydrostatic stresses show their dependency on coordinate axis and orientation of crystal with respect to the principal stress. However due to viscoelastic behavior of polymeric materials, most of the assumptions made for linear dielectric material are not valid for elastomer up to large extent for large deformation. Despite of these limitations, the researchers are frequently using the formula derived on assumptions made for a linear elastic and linear dielectric material.

\subsection{Boundary Conditions: Dependence of Stress Tensor on the Orientation of Dielectric Slab with Respect to the Electric Field Vector}

The expression (27) is valid if the direction of electric field $\underline{E}_{z}$ is making an angle $\theta$ with respect to the normal to the surface of sample (Figure 5), then the expression [3] for the stress tensor in triclinic case would be 


$$
T_{i j}=\left[\begin{array}{ccc}
-\frac{1}{2} \varepsilon_{33} E_{z}^{2} & 0 & 0 \\
\varepsilon_{13} E_{z}^{2} \sin \theta & \frac{1}{2} \varepsilon_{33} E_{z}^{2}\left(\sin ^{2} \theta-\cos ^{2} \theta\right)+\varepsilon_{23} E_{z}^{2} \sin \theta \cos \theta & \varepsilon_{33} E_{z}^{2} \sin \theta \cos \theta-\varepsilon_{23} E_{z}^{2} \sin \theta \\
\varepsilon_{13} E_{z}^{2} \cos \theta & \varepsilon_{33} E_{z}^{2} \sin \theta \cos \theta+\varepsilon_{23} E_{z}^{2} \cos ^{2} \theta & \frac{1}{2} \varepsilon_{33} E_{z}^{2}\left(\cos ^{2} \theta-\sin ^{2} \theta\right)-\varepsilon_{23} E_{z}^{2} \sin \theta \cos \theta
\end{array}\right]
$$

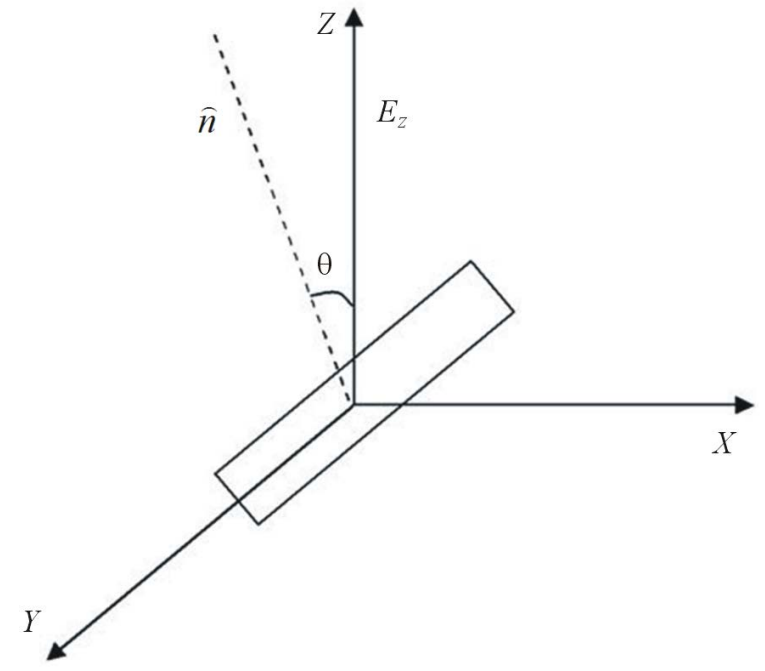

Figure 5. Schematic diagram illustrating the normal to the surface of a sample makes an angle $\theta$ with the direction of applied electric field $E$ i.e. orientation of sample with electric field vector.

For experimental study [6] [17], a parallel plate capacitor with dielectric film/slab has been considered due to its simple symmetrical geometry. However, due to electrostrictive deformation, the permittivity of different regions is different so the solution to the field or potential must be different although having the same general form of solution (to the Laplace equation). The appropriate electrical boundary conditions must be satisfied at the interface between two regions.

For mechanical boundary conditions, the external forces on the boundary may be regarded as a continuation of the internal stress distribution and in equilibrium, the total forces consisting of total internal body forces and total external forces, like forces due to gravitational field, in every volume element of elastic dielectrics must be balanced to a zero value [4].

However it is very difficult to predict the exact boundary conditions taking place at the interface between the electrodes and the deformed dielectrics or between two regions with different permittivities and non-uniform field. The interface in Nanometric dielectrics with non-uniform electric fields also exhibits electromechanical properties [19].

\section{Conclusion}

The idea of the dependence of electrostatic stresses on the orientation of dielectric material slab with respect to electric field vector is necessary for the correct measurement of electrically induced strains. Most of the recent works fail to correlate correctly the Maxwell stress tensor, the electrostrictive stress tensor and the mechanical stress tensor, as the assumptions made for their formulations are different. As the details of exact boundary conditions at the electrode-dielectric interface of a deformed elastic material are unknown, the correct estimation of electrically induced deformation is very difficult. Contribution due to lateral/shear components of dielectric constant as in Equation (27), in addition to the longitudinal deformation, must be incorporated into the mathematical expression to get correct estimation of results. In elastic dielectrics, the shear and lateral stresses play a major role and hence neglect of the contribution from lateral and shear stresses leads to over estimation or under esti- 
mation of results. Experimental data on the subject are not abundant, and the researchers [5] [6] frequently used an incorrect formula in the derivation of elastic strain with respect to the Maxwell stress effect, particularly in the case of polyurethane elastomer (a cross-linked polymer), assuming various unrealistic approximations.

\section{References}

[1] Landau, L.D. and Lifshitz, L.M. (1984) Electrodynamics of Continuous Media. 2nd Edition, Pergamon Press, Oxford.

[2] Thakur, O.P. and Singh, A.K. (2009) Electrostriction and Electromechanical Coupling in Elastic Dielectrics at Nanometric Interfaces. Materials Science-Poland, 27, 839-850.

[3] Kloos, G. (1995) The Dependence of Electrostatic Stresses at the Surface of a Dielectric on Its Orientation in an Electric Field. Journal of Physics D: Applied Physics, 28, 2424-2429. http://dx.doi.org/10.1088/0022-3727/28/12/006

[4] Stratton, J.A. (1941) Electromagnetic Theory. McGraw-Hill, New York.

[5] Shkel, Y.M. and Klingenberg, D.J. (1996) Material Parameter for Electrostriction. Journal of Applied Physics, 80, 4566. http://dx.doi.org/10.1063/1.363439

[6] Zhang, Q.M., Su, J., Kim, C.H., Ting, R. and Capps, R. (1997) An Experimental Investigation of Electromechanical Responses in a Polyurethane Elastomer. Journal of Applied Physics, 81, 2770. http://dx.doi.org/10.1063/1.363981

[7] Krakovský, I., Romijn, T. and Posthuma de Boer, A. (1999) A Few Remarks on the Electrostriction of Elastomers. Journal of Applied Physics, 85, 628. http://dx.doi.org/10.1063/1.369418

[8] Ladabaum, I., Khuri-Yakub, B.T. and Spoliansky, D. (1996) Micromachined Ultrasonic Transducers: $11.4 \mathrm{MHz}$ Transmission in Air and More. Applied Physics Letters, 68, 7. http://dx.doi.org/10.1063/1.116764

[9] Herbert, J. M. (1982) Ferroelectric Transducers and Sensors. Gordon and Breach, New York.

[10] Bar-Cohen, Y. (Ed.) (2004) Electroactive Polymer (EAP) Actuators as Artificial Muscles: Reality, Potential and Challenges. 2nd Edition, SPIE Press, Bellingham, Vol. PM136.

[11] Juretschke, H.J. (1977) Simple Derivation of Maxwell Stress Tensor and Electrostrictive Effect in Crystal. American Journal of Physics, 45, 277-280. http://dx.doi.org/10.1119/1.10642

[12] Lewis, T.J. (2005) Interfaces: Nanometric Dielectrics. Journal of Physics D: Applied Physics, 38, 202-212. http://dx.doi.org/10.1088/0022-3727/38/2/004

[13] Barletta, A. and Zanchini, E. (1994) Can the Definition of Mechanical Stress Tensor Be Applied to a Dielectric Fluid in an Electrostatic and Magneto Static Field? IL Nuovo Cimento, 16D, 177-187.

[14] Wang, C.C. (1979) Mathematical principles of Mechanics and Electromagnetism. Plenum, New York.

[15] Truesdell, C. (1966) The Elements of Continuum Mechanics. Springer-Verlag, Berlin.

[16] Kanno, I., Kotera, H. And Wasa, K. (2003) Measurement of Transverse Piezoelectric Properties of PZT Thin Films. Sensors and Actuators Actuators A, 107, 68-74.

[17] Lee, H.Y., Peng, Y. and Shkel, Y.M. (2005) Strain-Dielectric Response of Dielectrics as Foundation for Electrostriction Stresses. Journal of Applied Physics, 98, Article ID: 074104. http://dx.doi.org/10.1063/1.2073977

[18] Thakur, O.P. and Singh, A.K. (2008) Modeling of Capacitive Sensor Filled with Elastic Dielectrics and Its Advantages. 3rd International Conference on Sensing Technology, 30 November-3 December 2008, Taiwan, 467-471.

[19] Thakur, O.P. and Singh, A.K. (2007) Electromechanical Phenomena at the Interface in Nanometric Dielectrics. Proceedings of 2nd International Conference on Sensing Technology, 26-28 November 2007, Palmerston North, 188-192. 